EXTENDED REPORT

\title{
A randomised clinical trial comparing $2 \%$ econazole and $5 \%$ natamycin for the treatment of fungal keratitis
}

\author{
N V Prajna, R K John, P K Nirmalan, P Lalitha, M Srinivasan
}

See end of article for

authors' affiliations

Br J Ophthalmol 2003;87:1235-1237

Correspondence to: N Venkatesh Prajna, Cornea Service, Aravind Eye Care System, 1 Anna Nagar, Madurai-625020, India;

prajna@aravind.org

Accepted for publication 18 February 2003

\begin{abstract}
Aim: To compare $2 \%$ econazole and $5 \%$ natamycin in the management of fungal keratitis. Methods: A randomised clinical trial was performed using $2 \%$ econazole or $5 \%$ natamycin as the two treatment arms on patients presenting with culture positive fungal keratitis to the cornea service at Aravind Eye Care System, Madurai, India.

Results: 116 patients were recruited, and 112 continued in the study. There were no significant differences between the two arms at baseline or for success (defined as a healed or healing ulcer) at final visit $(p=0.79)$.

Conclusions: $2 \%$ Econazole appears to be as effective as $5 \%$ natamycin for the management of fungal keratitis.
\end{abstract}

orneal scarring, either as a result of trauma or corneal infections, is recognised as a major cause for vision impairment and blindness..$^{1-5}$ There is wide geographical variation in the epidemiological pattern of corneal ulcers across and even within countries. ${ }^{6}$ Recent reports suggest an increasing prevalence for fungal ulcers in developing countries. ${ }^{6-8}$ A previous study reported the incidence of corneal ulcers in Madurai district of southern Tamil Nadu in India to be 113 per 100000 population (approximately 10 times the incidence reported from the United States). ${ }^{\circ}$ A study conducted by Srinivasan et al reported fungal keratitis as an enormous public health problem in south India. ${ }^{6}$ Fungi, especially Fusarium (47\%) and Aspergillus (16\%), were identified as the aetiological agents responsible for $44 \%$ of all corneal ulcers in that study. ${ }^{6}$ Previous reports from Ghana ${ }^{7}$ and northern Tanzania ${ }^{8}$ have reported fungi as the aetiological agent in over $50 \%$ of culture positive cases of keratitis. The aetiological agent for fungal keratitis may vary within countries-Fusarium species is more commonly associated with fungal keratitis in southern India, ${ }^{7}$ while Aspergillus species is more often implicated in northern India and adjoining Nepal. ${ }^{10}$ Besides Fusarium and Aspergillus, a large number of demataceous fungi, filamentous fungi, yeasts, and other dimorphic organisms have been implicated as aetiological agents for fungal keratitis. Until recently, only a single drug was available for the management of fungal keratitis in developing countries despite the known potential for vision impairment and blindness associated with fungal keratitis, ${ }^{8}$ and the large number of fungal species identified with keratitis. We designed a randomised clinical trial to compare the efficacy and safety of $2 \%$ topical econazole with $5 \%$ topical natamycin for the management of fungal keratitis.

\section{METHODS}

The patients for this study were recruited from the cornea service of Aravind Eye Care System, Madurai. Each patient with keratitis who presented to the department had a clinical examination using a slit lamp biomicroscope to measure size and depth of ulceration following a detailed clinical and demographic history. Scraping of the corneal ulcer using a Kimura spatula was performed under magnification. The material obtained from the initial scraping was subjected to Gram stain evaluation to identify bacteria, and $10 \%$ potassium hydroxide mount to identify fungus. Subsequent scrapings were plated onto blood agar and potato dextrose agar. Patients with proved fungal keratitis (smear and culture positive for fungus) with an ulcer area of at least $2 \mathrm{~mm}^{2}$ and not more than $60 \mathrm{~mm}^{2}$ were identified as potential participants for the study. Patients who were not willing to be part of the study, and who did not meet entry criteria were excluded from the study.

After obtaining necessary informed consent subjects were randomised to receive either $2 \%$ econazole or $5 \%$ natamycin. Since natamycin is available as a suspension, and precipitates in the corneal tissue, it was not possible to mask the investigator to the drugs used on subsequent visits. All participating subjects were admitted to the wards for a week, and had either $2 \%$ econazole or $5 \%$ natamycin eye drops instilled on an hourly basis between $7 \mathrm{am}$ and $9 \mathrm{pm}$. Additionally, 1\% atropine sulphate ointment was applied three times per day in the affected eye at least 15 minutes after application of the antifungal eye drops. The clinical investigator examined each subject on a daily basis using a slit lamp biomicroscope, and measured the size and depth of the infiltration.

Subjects were discharged from the hospital at the end of the first week with instructions to instil the eye drops in the affected eye at intervals of 2 hours between 7 am and 9 pm. Each subject was examined at the slit lamp for size and depth of the infiltrate at weekly intervals for up to 4 weeks after entry into the study. Exit criteria from the study were determined as a clinical worsening of the ulcer-if the size and depth of the infiltrate had increased by at least $20 \%$ with respect to the previous visit or perforation-or adverse reactions to the drops. Additionally, details of signs including lid oedema, congestion of the conjunctiva, and hypopyon were recorded for each subject. The presence or absence of hypopyon in the anterior chamber was noted, and quantified in millimetres.

We defined a healed corneal ulcer as a completely healed epithelial defect with no stain on fluorescein application, and non-progression of the stromal infiltration. A corneal ulcer was considered to be healing if the epithelial defect decreased in size by at least $20 \%$, with non-progression or decrease in the size of the stromal infiltration by at least $20 \%$. A corneal ulcer was considered to remain the same if the size and depth of the infiltrate remained same after initiating treatment. An 
ulcer was considered to worsened if the size and depth of the ulcer increased by at least $20 \%$, or if the ulcer perforated.

Success of the treatment was defined as a healed or healing ulcer at final visit, and failure as an ulcer that worsened or remained same at final visit. We analysed the time to event (success) for comparing the efficacy of the two medications, and the influence of the medications on signs including lid oedema, congestion, and hypopyon was taken as the measure to compare safety of the medications. Tests for significance included $t$ test to compare the means of continuous variables, Pearson $\chi^{2}$ and Fisher's exact test for categorical variables, log rank test for the mean of survival rates, and Kaplan-Meier procedure to estimate survival rate at each point of time with a healed or healing ulcer considered as an event.

\section{RESULTS}

We recruited and randomised 116 culture positive eyes of 116 patients of fungal keratitis who presented to the cornea clinic from March to October 2002, to either 2\% econazole or 5\% natamycin. Four of the 116 patients randomised at baseline did not return for further follow up (Fig 1) and were dropped from the study. All these cases were males, and were equally distributed between econazole and natamycin.

The mean age of subjects was 37.0 years (SD 13.8, median 36.0 years, range $7.0-84$ years), and $72(64.3 \%)$ were males. Fifty nine $(52.7 \%)$ subjects were randomised to econazole and $53(47.3 \%)$ to natamycin. There was no difference in age $(p=0.43)$ or sex $(p=0.70)$ between those randomised to receive either econazole or natamycin. There was no significant difference at baseline in either size of epithelial defect $(p=0.89)$, size of infiltrate $(p=0.95)$, or depth of infiltrate $(p=0.24)$ between the two arms of the study. There was no significant difference in lid oedema $(p=0.22)$, congestion $(p=0.78)$, or hypopyon $(p=1.0)$ between the two arms at baseline. Organisms responsible for fungal keratitis were also not significantly different between the two groups (see Table 1).

There was no significant difference (log rank 0.52, $\mathrm{p}=0.47$ ) between the two arms for success. There was no significant difference in the time to heal based on baseline size of epithelial defects ( $\log$ rank 0.82, $\mathrm{p}=0.37$ ), size of infiltrate ( $\log$ rank $0.86, \mathrm{p}=0.35$ ) or depth of infiltrate (log rank $0.74, p=0.39$ ) between the two arms of the study. There was no difference in the time to subside for signs including lid oedema $(\log \operatorname{rank} 1.05, \mathrm{p}=0.31)$, congestion of the conjunctiva ( $\log$ rank $0.51, \mathrm{p}=0.47$ ) or hypopyon (log rank $0.23, p=0.63$ ) between the two arms. Figure 2 shows the Kaplan-Meier survival curves for the two arms based on time taken to heal in days.

\section{DISCUSSION}

Although multiple randomised clinical trials are performed on a regular basis to address bacterial keratitis, there are few randomised clinical trials addressing fungal keratitis. ${ }^{11}{ }^{12}$ Data from our study suggest that $2 \%$ econazole can be used as an alternative to $5 \%$ natamycin in the management of fungal keratitis.

The use of imidazoles as antifungal agents has been reported previously. ${ }^{13-15}$ Two small case series have also reported use of econazole as a useful agent in the management of fungal keratitis. ${ }^{16}{ }^{17}$ Natamycin is currently considered the most effective medication against Fusarium and Aspergillus, ${ }^{18}$ which are responsible for most fungal keratitis, and until recently was the only topical antifungal agent available in India. Previous studies have reported poor outcomes for patients with fungal keratitis with one study reporting evisceration in $25 \%$ of patients suggesting that current treatments may not be very effective. ${ }^{8}$

Natamycin binds preferentially to ergosterol on the fungal plasma membrane and causes localised membrane disrup-

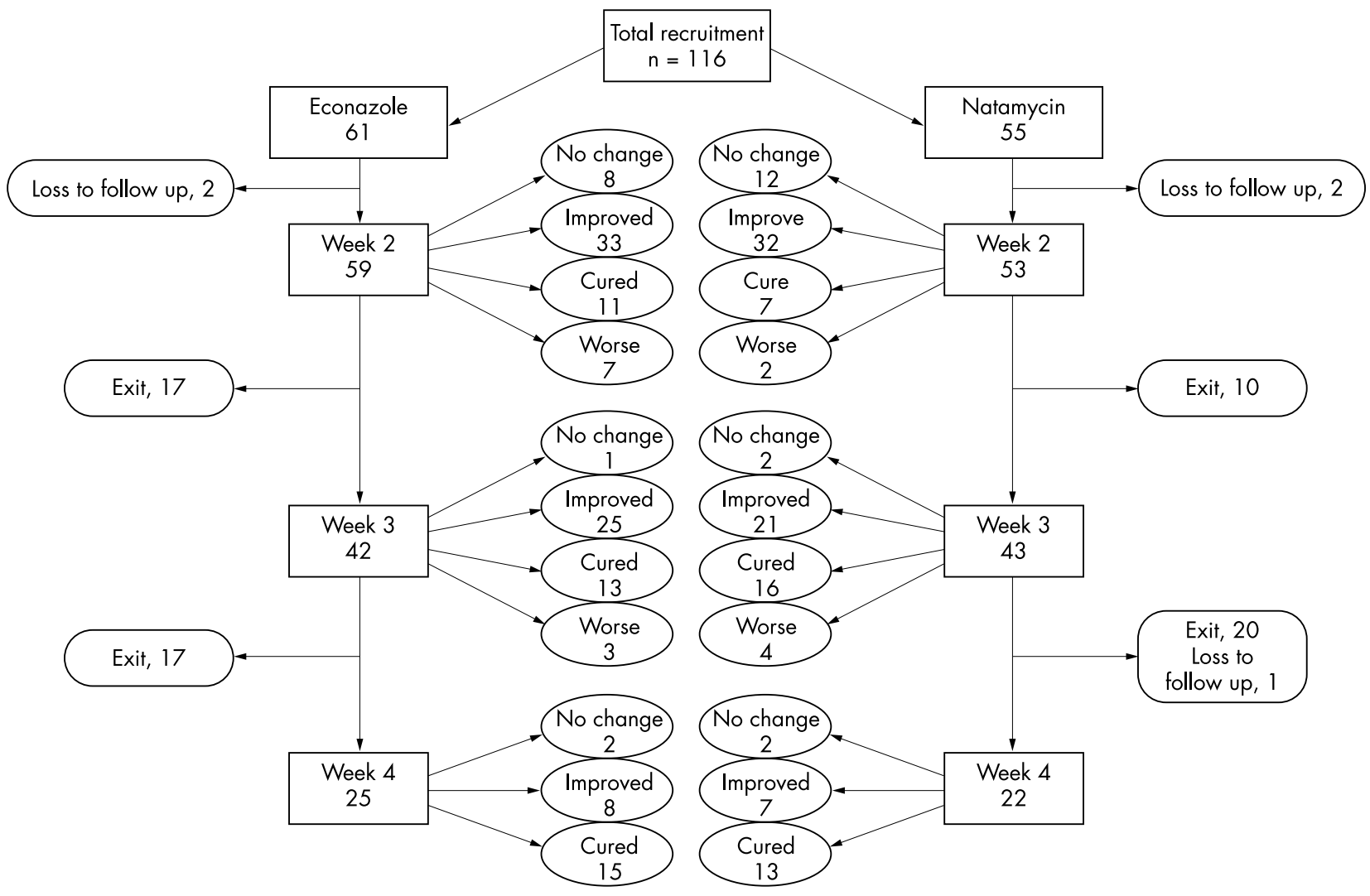

Figure 1 Flow chart of the participation in the study. 
Table 1 Aetiological organisms of fungal keratitis by treatment arm

\begin{tabular}{lccc}
\hline Organism & Econazole & Natamycin & Total \\
\hline Aspergillus flavus & $10(16.9)$ & $11(20.7)$ & $21(18.7)$ \\
Aspergillus fumigatus & $3(5.1)$ & $2(3.8)$ & $5(4.5)$ \\
Aspergillus niger & $3(5.1)$ & $1(1.9)$ & $4(3.6)$ \\
Cladosporium & $1(1.7)$ & $1(1.9)$ & $2(1.8)$ \\
Curvularia & $3(5.1)$ & $3(5.7)$ & $6(5.4)$ \\
Fusarium species & $32(54.2)$ & $32(60.4)$ & $64(57.1)$ \\
Unidentified hyaline & $7(11.9)$ & $3(5.7)$ & $10(8.9)$ \\
Total & $59(100.0)$ & $53(100.0)$ & $112(100.0)$ \\
\hline
\end{tabular}

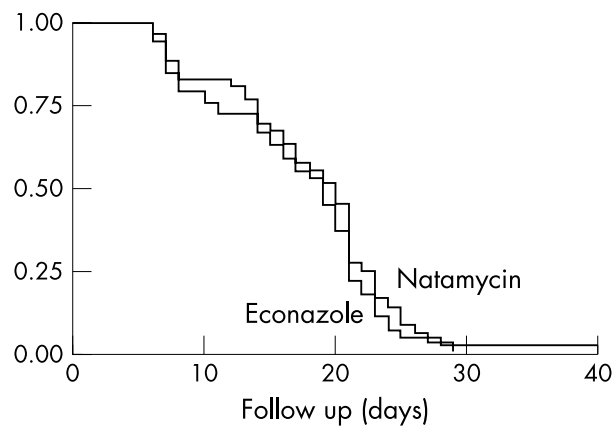

Figure 2 Survival curves comparing 2\% econazole and 5\% natamycin for fungal keratitis.

tions by altering membrane permeability. ${ }^{19}$ The imidazoles exhibit antifungal activity through two mechanisms of action depending on the concentration. At lower concentrations, they are fungistatic and inhibit sterol-4-alpha demethylase, a microsomal P450 related enzyme, ${ }^{20}$ while they are fungicidal at higher concentrations causing direct membrane damage to the phospholipids present in the fungal cell wall. ${ }^{21}$ It is possible that use of both natamycin and econazole in the management of fungal keratitis may improve chances for more rapid resolution of corneal ulcers, especially since the mechanisms of action are different. However, further studies are required to validate this hypothesis.

While means to identify aetiological agents are available, the lack of effective antifungal agents often translates to an inability to treat the keratitis despite diagnosis. However, currently, fungal keratitis does not appear to be a priority or focus for pharmaceutical companies that apparently prefer to develop new lines of antibiotics against bacterial keratitis, which is more prevalent in the developed world.

\section{ACKNOWLEDGEMENTS}

Aravind Medical Research Foundation, Madurai, supported the study.

\section{Authors' affiliations}

N V Prajna, R K John, P K Nirmalan, P Lalitha, M Srinivasan, Aravind Eye Care System, Madurai, India

R K John, P K Nirmalan, Lions Aravind Institute of Community Ophthalmology, Madurai, India

Financial interest: None

\section{REFERENCES}

1 Chirambo MC, Tielsch JM, West KP, et al. Blindness and visual impairment in southern Malawi. Bull World Health Organ 1986;64:567-72.

2 Rapoza PA, West SK, Katala SJ, et al. Prevalence and causes of vision loss in central Tanzania. Int Ophthalmol 1991;15:123-9.

3 Brilliant LB, Pokhrel RP, Grasset NC, et al. Epidemiology of blindness in Nepal. Bull World Health Organ 1985;63:375-86.

4 Khan MU, Hague MR, Khan MR. Prevalence and causes of blindness in rural Bangladesh. Ind J Med Res 1985;82:257-62.

5 Gilbert CE, Wood M, Waddel K, et al. Causes of childhood blindness in East Africa: results in 491 pupils attending 17 schools for the blind in Malawi, Kenya and Uganda. Ophthalmic Epidemiol 1995;2:77-84.

6 Srinivasan M, Gonzales CA, George C, et al. Epidemiology and aetiological diagnosis of corneal ulceration in Madurai, south India. Br J Ophthalmol 1997;81:965-71

7 Hagan M, Wright E, Newman M, et al. Causes of suppurative keratitis in Ghana. Br J Ophthalmol 1995:79:1024-8.

8 Poole TRG, Hunter DL, Maliwa EMK, et al. Aetiology of microbial keratitis in nothern Tanzania. Br J Ophthalmol 2002;86:941-2.

9 Gonzales CA, Srinivasan M, Whitcher JP, et al. Incidence of corneal ulceration in Madurai District, south India. Ophthalmic Epidemiol 1996;3:159-66.

10 Upadhyay MP, Karmacharya PC, Koirala S, et al. Epidemiology characteristics, predisposing factors and etiologic diagnosis of corneal ulceration in Nepal. Am J Ophthalmol 1991;111:92-9.

11 O'Brien, Maguire MG, Fink NE, et al. Efficacy of oflaxacin vs cefazolin, and tobramycin in the therapy for bacterial keratitis: report from the Bacterial Keratitis Study Research Group. Arch Ophthalmol 1995;1 13:1257-65.

12 Prajna NV, George C, Selvaraj S, et al. Bacteriologic and clinical efficacy ofloxacin $0.3 \%$ versus ciprofloxacin $0.3 \%$ ophthalmic solution in the treatment of patients with culture-positive bacterial keratitis. Cornea 2001;20:175-8.

13 Foster CS. Miconazole therapy for keratomycosis. Am J Ophthalmol 1981;91:622-9.

14 Fitzsimons R, Peters AL. Miconazole and ketoconazole as a satisfactory first line treatment for keratomycosis. Am J Ophthalmol 1986;101:605-8.

15 Dupont B, Drouhet E. Early experience with itraconazole in vitro and in patients: pharmokinetic studies and clinical results. Rev Infect Dis 1987:9:571-6

16 Arora I, Kulshrestha OP, Upadhaya S. Treatment of fungal corneal ulcers with econazole. Ind J Ophthalmol 1983;31:1019-21.

17 Mahashabde S, Nahata MC, Shrivastava U. A comparative study of antifungal drugs in mycotic corneal ulcer. Ind J Ophthalmol 1987;35:149-52.

18 O'Day DM. Selection of appropriate antifungal therapy. Cornea 1987:6:238-45.

19 Medoff G, Kobayaski GS. Strategies in the treatment of systemic fungal infections. N Engl J Med 1980;302:145-55.

20 Sud IJ, Feingold DS. Mechanism of action of the anti-mycotic imidazoles. $J$ Invest Dermatol 1981;6:438-41.

21 Beggs WH, Hughes CE. Exploitation of the direct cell damaging action of antifungal azoles. Diagn Microbiol Infect Dis 1987;6:1-3. 